Rev. salud pública. 13 (4): 597-609, 2011

\title{
Inactividad física y factores de riesgo: aproximación a un modelo interpretativo para Bogotá
}

\section{Physical inactivity and risk factors: constructing an explanatory model in Bogota}

\author{
Xiomara Uribe-Bustos ${ }^{1}$ y Carlos Agudelo-Calderón ${ }^{2}$ \\ 1 Secretaría de Salud de Cundinamarca. Bogotá, Colombia. xiomarauribe@gmail.com. \\ 2 Instituto de Salud Pública. Facultad de Medicina. Universidad Nacional de Colombia. \\ caagudelo@unal.edu.co
}

Recibido 23 Junio 2010/Enviado para Modificación 20 Abril 2011/Aceptado 25 Julio 2011

\section{RESUMEN}

Objetivo Determinar los factores asociados con la inactividad física en adultos de 25 a 50 años de Bogotá y aproximarse a la definición de un modelo explicativo. Materiales y Métodos Se utilizó una muestra de 1600 adultos caracterizados en 2004 con el IPAQ largo y se midió la prevalencia de actividad física global y por dominios. Se determinaron los factores asociados utilizando regresión logística. Resultados Se encontró $43,8 \%$ de adultos inactivos, $55,6 \%$ en el dominio de transporte, $53,6 \%$ en el hogar, $48,8 \%$ en tiempo libre y $32,2 \%$ en el trabajo. El modelo incluyó como factores asociados ocuparse los últimos 7 días en trabajar, trabajar y estudiar, edad entre 25 y 40 años y percepción del estado de salud muy bueno. En el dominio del transporte, estado de salud muy bueno. En el dominio del hogar, ser mujer, ningún nivel educativo preescolar y secundaria, ocuparse en labores del hogar; estado de salud muy bueno, bueno o regular. En el dominio de tiempo libre, no tener una pareja estable, no fumar y observar la presencia de personas físicamente activas en el barrio. $Y$ en el dominio del trabajo ocuparse en trabajar, y trabajar y estudiar.

Conclusión Los factores asociados a la actividad física global y por dominios, permiten comprender de manera más apropiada los determinantes específicos del nivel de actividad física.

Palabras Clave: Actividad motora, adulto, salud urbana, promoción de la salud, Colombia (fuente: DeCS, BIREME).

\section{ABSTRACT}

Objective Determining factors associated with the 25-50 year-old population's physical inactivity in Bogota, Colombia (urban area) and, thereby, defining an explanatory model. 
Methods A sample of 1,600 adults which had been characterised in 2004 using the long international physical activity questionnaire (IPAQ-L) was used; overall physical activity prevalence was measured and discriminated by domain. Logistic regression models were used for determining associated factors.

Results This research found that $43.8 \%$ of the adult population were inactive $(55.6 \%$ regarding mode of transport, $53.6 \%$ the home, $48.8 \%$ leisure time and $32.2 \%$ being work-related). The model had related factors such as working during the past seven days, working, studying and being aged 25 to 40 years old and perception of leading a healthy life. Domestic activities were related to being a woman and educational level (no preschool and/or high school education) regarding very good, good and regular state of health. Leisure time was related to being physically active, not having a stable partner, not smoking and observing that other people were physically active in their home area. Hard work and combining study and work were associated with the workrelated domain.

Conclusions Future public healthcare action should be based on factors associated with overall physical activity and domains, thereby allowing the most appropriate specific physical activity determinants to be understood.

Key Words: Motor activity, adult, urban health, healthcare promotion, Colombia (source: $\mathrm{MeSH}, \mathrm{NLM}$ ).

L as enfermedades crónicas no transmisibles (ECNT) son motivo de preocupación en Colombia debido a la gran carga de morbi-mortalidad incapacidad en la población. Durante el periodo 1990-1996 la hipertensión arterial, diabetes y problemas cardiacos representaron el 44,5\% del total de la mortalidad, siendo uno de los principales factores de riesgo el sedentarismo (1), el cual puede modificarse con intervenciones que promuevan estilos de vida saludables como la actividad física, cuyos beneficios son considerables en la prevención de las ECNT (2-9).

En el año 2003, el Ministerio de la Protección Social y Colciencias, adelantaron una convocatoria para llevar a cabo una investigación dirigida al fomento de estilos de vida saludables con énfasis en actividad física. Como respuesta, la Universidad Nacional en unión con la Fundación Ciudad Humana, presentaron el proyecto "Modelos de Movilización Social con énfasis en la actividad Física y estilos de vida saludables para reducir el sedentarismo en las regiones de Bogotá D.C., Antioquia y Quindío" (10). Se ejecutó durante el año 2004 y encontró alta prevalencia de inactividad física por dominios para Bogotá: $76 \%$ en tiempo libre, $99.8 \%$ en el interior hogar, $86 \%$ en el exterior de la casa y $78 \%$ como parte del trabajo. A partir de la línea de base de éste estudio se diseñó el trabajo analítico cuyos resultados se presentan en éste artículo. 
Otros estudios reportaron para Bogotá niveles de inactividad física globales en población de 18-64 años entre 55,3 \% (5) y 63,2 \% (12), y 19,8 \% (13) con población mundial estandarizada de 2002. También se ha reportado prevalencias de inactividad física elevadas por dominios. Mantilla (11) encontró $59 \%$ en tiempo libre, $55 \%$ como parte del trabajo, $54 \%$ en el dominio del hogar y $60 \%$ como medio de transporte. ENSIN (5) encontró $74,8 \%$ en el dominio de caminar como medio de transporte, $72,3 \%$ como parte del trabajo y $80,5 \%$ en el hogar.

Se ha encontrado como factores de riesgo asociados a la inactividad física ser mujer y bajo nivel socioeconómico $(2,4,6,12-15)$; nivel educativo elevado $(5,11,16)$; ocupación hogar, buscar trabajo y estudiar $(5,12)$; auto-percepción del estado de salud bueno y muy bueno (12); caminar con el soporte social (8), y percepción de inseguridad durante la noche (11). Además, se ha encontrado disminución de la actividad física a partir de la adolescencia que continúa a lo largo de la vida $(2,6,13,17)$. En Bogotá, se encontró como factores asociados a la actividad física ser hombre, personas con autopercepción del estado de salud buena o muy buena, edad entre 30 y 65 años -en hombres- y actividad principal en el último mes buscar trabajo o realizar labores en el hogar (12).

A pesar de lo descrito, el conocimiento es limitado para tomar decisiones respecto a las intervenciones más ajustadas a las condiciones de la población de Bogotá, por ello, en presente artículo describe los factores asociados a la inactividad física global y por dominios, intentando explicar mediante modelos éste comportamiento.

\section{MÉTODOS}

A partir de una encuesta transversal de línea de base del estudio Modelos de Movilización Social con Énfasis en la Actividad Física y Estilos de Vida Saludables para Reducir el Sedentarismo en la Región de Bogotá D.C. $(10,18)$, se realizó un ejercicio de modelamiento para determinar los factores asociados y definir un modelo explicativo de la inactividad física en adultos de 25 a 50 años, de la zona urbana de Bogotá D.C. Se utilizó la muestra de 1 600 personas adultas, estimada en el año 2004 como representativa de la población de estrato 3 de Bogotá, con $50 \%$ de proporción del régimen contributivo, $24 \%$ de actividad física en adultos, precisión del $2 \%$, intervalo de confianza del $99 \%$ y seleccionada en las localidades de Tunjuelito, Kennedy, Suba y Rafael Uribe Uribe de Bogotá. La información utilizada se había 
recolectado con el Cuestionario Internacional de Actividad Física (IPAQ) versión larga en español. Se calculó la prevalencia de actividad física global en las categorías baja, moderada y alta, y en los dominios tiempo libre, trabajo, medio de transporte, y actividades domésticas y en el jardín. El procesamiento de los datos se realizó utilizando la Guía para el procesamiento de datos y análisis del IPAQ (19). También se retomó la información del módulo de Contexto urbano con el cual se evaluó la asociación de las categorías de la actividad física con aspectos del entorno. Como apoyo informático se utilizó Excel y SPSS versión 11.5.

Los criterios que se tuvieron en cuenta para clasificar a los individuos por categorías alta, moderada y baja corresponden a la recomendación de salud pública mundial $(4,20,21)$ incluyendo los individuos que no reportan actividad física dentro de la categoría baja.

En cuanto a los dominios, se consideró que cumplen con la actividad física recomendada en tiempo libre las personas que realizan diariamente al menos 30 minutos de actividad física en esfuerzos acumulados de 10 minutos seguidos por al menos 5 días a la semana $(4,20,21)$. Esta misma definición operativa se utilizó para los dominios trabajo, hogar y medio de transporte. De esta manera se clasificó a los individuos en dos categorías: inactivos y cumple con la actividad física recomendada.

Las categorías globales y los dominios se cruzaron con las variables socio-demográficas, percepción del estado de salud, estilo de vida y contexto urbano. Se calcularon intervalos de confianza y significación estadística con la prueba chi cuadrado ( $\mathrm{p} \leq 0.05)$, con lo cual se identificó los factores individualmente asociados a la suficiencia de la actividad física.

El modelamiento se realizó mediante regresión logística, para lo cual se siguió los principios metodológicos descritos en la literatura (22-24). En consecuencia se recodificó la variable actividad física en dos categorías, actividad física suficiente que incluyó los niveles de actividad física moderada y alta, e inactivos o con actividad física insuficiente o baja. Se introdujeron las variables al modelo de regresión utilizando el método introducir del SPSS y al final se evaluó los cinco modelos con las medidas de bondad de ajuste.

El control del error aleatorio se realizó verificando los criterios probabilísticos utilizados para el cálculo del tamaño de la muestra descritos en el estudio Modelos de Movilización Social (10), y a través del cálculo en el 
presente estudio del poder para la muestra total que fue de $97.4 \% \mathrm{y}$ para cada uno de los dominios, siendo superior al $80 \%$, por lo que se concluye que el tamaño de la muestra permitió encontrar diferencias significativas cuando estas existían.

\section{RESULTADOS}

El estudio reveló 43,9 \% de actividad física baja, 37,9\% moderada y 18,3\% alta. Se presentó mayor prevalencia de inactividad física en mujeres, en personas entre 41 y 50 años, con pareja estable, con nivel de estudios primario y secundario, dedicados en los últimos 7 días a oficios del hogar y a buscar trabajo, con auto percepción del estado de salud deficiente, no fumadores actuales, y que no ingirieron bebidas alcohólicas en la última semana. Todas las variables fueron estadísticamente significativas (Tabla 1). También fueron significativas las variables de contexto urbano relacionadas con infraestructura como existencia de instalaciones de recreación sin costo o de bajo costo, presencia de cosas agradables para mirar mientras camina, presencia de cruces viales peligrosos en el barrio y alrededor, sitios para montar en bicicleta cotidianamente (ciclo rutas todos los días) bien mantenidos y sin obstrucciones, y la variable de soporte social se observa la presencia de personas físicamente activas.

El modelo explicativo de la actividad física global incluyó como variables asociadas al cumplimiento de la recomendación: trabajar y trabajar-estudiar como actividad a la que la persona se dedicó la mayor parte del tiempo durante los últimos 7 días, tener entre 25 y 40 años, tener muy buena salud, en tanto que no fumar se asoció inversamente con ser activo físicamente (Tabla 2).

En los dominios se encontraron las mayores prevalencias de inactividad física en el dominio de la actividad física como medio de transporte seguida de hogar y tiempo libre, y la menor prevalencia se encontró en el dominio del trabajo (Tabla 3). En éste último se hallaron asociadas las variables sexo, actividad los últimos 7 días y fumar actualmente, y el modelo explicativo incluyó como factores asociados de manera inversa ser mujer $\mathrm{OR}=0,672$ IC95 \% $(0,51-0,885)$ y no fumar OR=0,648 IC95 \% $(0,477-0,880)$. 


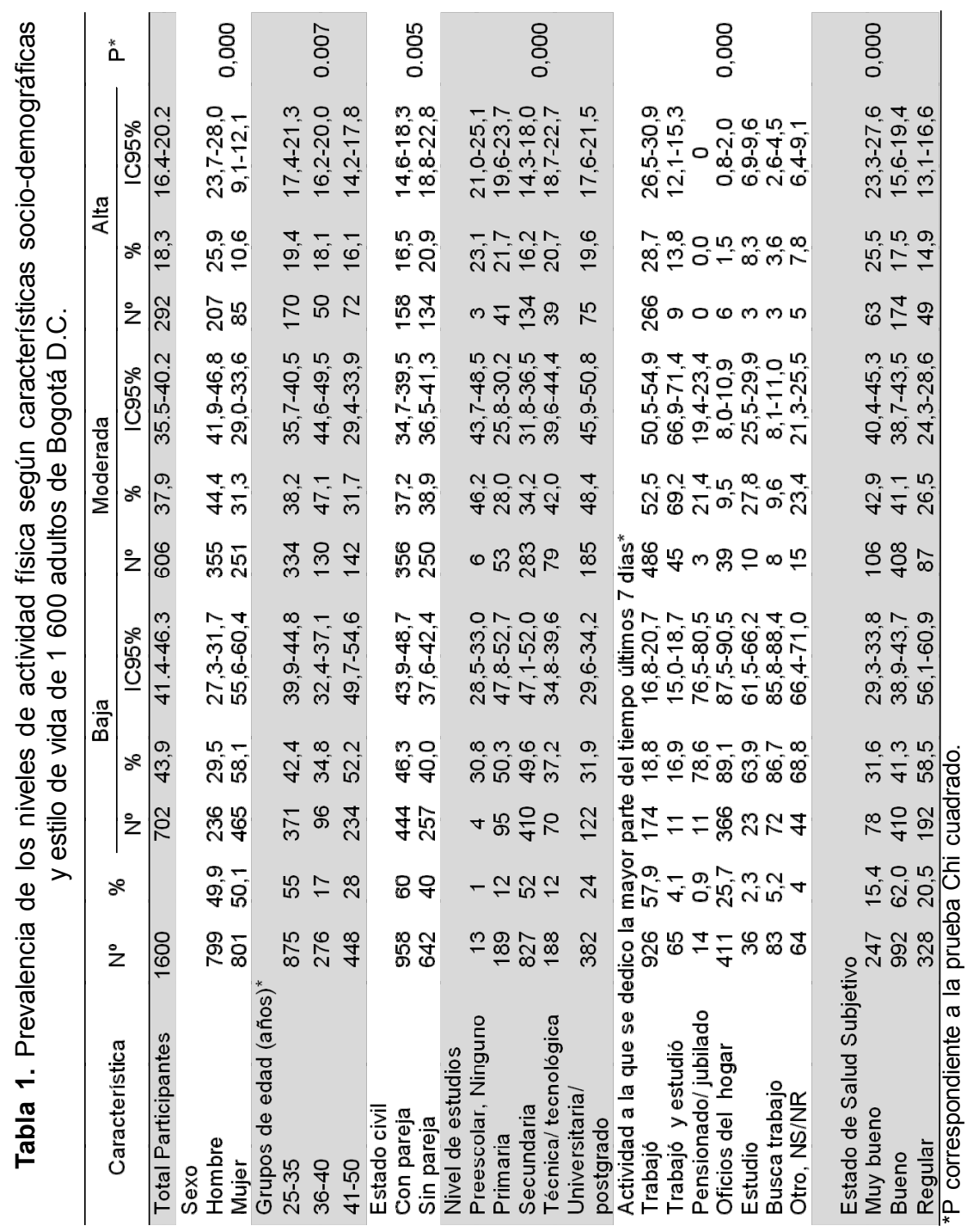


Tabla 2. Variables explicativas de la actividad física en adultos de 25 a 50 años en Bogotá D.C.

\begin{tabular}{|c|c|c|c|}
\hline \multirow[b]{2}{*}{ Variables } & \multirow[b]{2}{*}{ OR } & \multicolumn{2}{|c|}{ IC $95 \%$} \\
\hline & & $\begin{array}{l}\text { Límite } \\
\text { inferior }\end{array}$ & $\begin{array}{l}\text { Límite } \\
\text { superior }\end{array}$ \\
\hline \multicolumn{4}{|l|}{ Actividad en los últimos 7 días } \\
\hline Estudió & 0 & & \\
\hline Trabajó & 9.089 & 4.228 & 19.538 \\
\hline Trabajó y estudió & 10.839 & 3.784 & 31.046 \\
\hline Pensionado/jubilados, labores del hogar y buscar trabajo & 0.300 & 0.135 & 0.663 \\
\hline \multicolumn{4}{|l|}{ Edad } \\
\hline 41 y 50 años & 0 & & \\
\hline 25 y 35 años & 1.543 & 1.095 & 2.173 \\
\hline 36 y 40 años & 1.768 & 1.136 & 2.754 \\
\hline \multicolumn{4}{|l|}{ Estado de salud } \\
\hline Regular y malo & 0 & & \\
\hline Muy bueno & 1.672 & 1.031 & 2.711 \\
\hline Bueno & 1.418 & 0.988 & 2.036 \\
\hline \multicolumn{4}{|l|}{ Fuma } \\
\hline No Fuma & 0.664 & 0.474 & 0.928 \\
\hline
\end{tabular}

Tabla 3. Prevalencia de individuos que cumplen con la actividad física recomendada e inactivos, global y por dominios de actividad física Bogotá D.C.

\begin{tabular}{|c|c|c|c|c|c|c|c|c|c|}
\hline \multirow{3}{*}{ Dominio } & \multirow{3}{*}{$\begin{array}{c}\text { Total } \\
\text { participantes } \\
\mathrm{N}^{\circ} \\
\end{array}$} & \multirow{2}{*}{\multicolumn{2}{|c|}{$\begin{array}{l}\text { Cumplen con la } \\
\text { actividad física* }\end{array}$}} & \multicolumn{2}{|c|}{ IC $95 \%$} & & & \multicolumn{2}{|c|}{ IC $95 \%$} \\
\hline & & & & \multirow{2}{*}{$\begin{array}{l}\text { Límite } \\
\text { inferior }\end{array}$} & \multirow{2}{*}{$\begin{array}{l}\text { Límite } \\
\text { superior }\end{array}$} & \multicolumn{2}{|c|}{ Inactivos } & \multirow{2}{*}{$\begin{array}{l}\text { Límite } \\
\text { inferior }\end{array}$} & \multirow{2}{*}{$\begin{array}{l}\text { Límite } \\
\text { superior }\end{array}$} \\
\hline & & $\mathrm{N}^{\circ}$ & $\%$ & & & $\mathrm{~N}^{\circ}$ & $\%$ & & \\
\hline Global & 1600 & 898 & 56,1 & 53,7 & 58,6 & 702 & 43,9 & 41,4 & 46,3 \\
\hline Medio de Transporte & 1560 & 692 & 44,4 & 41,9 & 46,9 & 869 & 55,7 & 53,2 & 58,2 \\
\hline Hogar & 1596 & 741 & 46,4 & 44 & 48,8 & 856 & 53,6 & 51,2 & 56 \\
\hline Tiempo libre & 484 & 248 & 51,2 & 46,7 & 55,7 & 237 & 49 & 44,5 & 53,5 \\
\hline Trabajo & 1000 & 678 & 67,8 & 64,9 & 70,7 & 322 & 32,2 & 29,3 & 35,1 \\
\hline
\end{tabular}

En el dominio medio de transporte se encontraron asociadas las variables sexo, estado civil, nivel de estudios y estado de salud, y el modelo explicativo incluyó auto-percepción del estado de salud muy bueno OR=2,763 IC95 \% (1,177$6,487)$ y de manera inversa no tener pareja estable $\mathrm{OR}=0,808$ IC $95 \%(0,658$ $0,992)$.

En el dominio del hogar se encontró asociado sexo, edad, nivel de estudios, actividad durante los últimos 7 días, consumo de alcohol, fumar actualmente y la pregunta de contexto ¿Hay muchos lugares distantes de su casa para caminar? El modelo explicativo incluyó como variables asociadas ser mujer OR=4,14 IC95 $\%(3,21-5,34)$, tener como formación ninguna/preescolar OR=5,9 IC95\% (1,42$24,44)$ y secundaria $\mathrm{OR}=1,39$ IC95 \% $(1,03-1,87)$, realizar oficios del hogar durante los últimos 7 días OR=3,12 IC95\% $(1,8-5,4)$, auto-percepción del estado de salud buena OR=3,43 IC95 \% $(1,41-8,29)$, muy buena OR=4,98 IC95\% $(1,98-12,53)$ y regular OR=3,45 IC95 \% $(1,4-8,49)$, y asociación inversa con edad menor a 40 años, siendo las personas entre 36 a 40 años (OR=0,3 IC95\% 
$(0,737-0,52))$ y 25 a 35 años $(\mathrm{OR}=0,3$ IC95 \% $(0,737-0,52))$ las que tienen mayor probabilidad de cumplir con la recomendación de actividad física.

En el dominio tiempo libre, se encontró asociación con estado civil, nivel de estudios y actividad a la que se dedicó los últimos 7 días. El modelo incluyó como variables explicativas asociadas no estar unido a una pareja estable $\mathrm{OR}=1,6 \mathrm{IC} 95 \%(1,07-2,37)$, no fumar actualmente OR=1,53 IC95 \% $(1,01$ $2,32)$, estar completamente $(\mathrm{OR}=2,17$ IC95 \% $(1,06-4,47))$ y parcialmente $(\mathrm{OR}=2,74$ IC95 \% $(1,25-6,01))$ de acuerdo con observar muchas personas físicamente activas en el barrio. Se encontró asociado de manera inversa el nivel de formación primaria $\mathrm{OR}=0,34 \mathrm{IC} 95 \%(0,155-0,783)$, estar parcialmente de acuerdo $(\mathrm{OR}=0,41$ IC95 \% $(0,235-0,716))$ y en desacuerdo $(\mathrm{OR}=0,322$ IC95 \% $(0,172-0,6))$, o no estar ni de acuerdo ni en desacuerdo $(\mathrm{OR}=0,371$ IC95 \% $(0,168-0,819)$ en que usualmente hay mucho tráfico en las calles que hace difícil o poco agradable caminar en el barrio.

\section{DISCUSIÓN}

Los hallazgos indican que el 43,9\% de los adultos de Bogotá son inactivos físicamente, en tanto que la ENSIN (5) reportó 55,3\%, diferencias atribuidas al rango de edad y al criterio de inclusión de individuos del régimen contributivo y estrato socioeconómico 3.

En el dominio del hogar se encontró una prevalencia de inactividad física del 56,3\%, en contraste la ENSIN reportó $80,5 \%$ y Mantilla $54 \%$ (11). La similitud con la prevalencia reportada por Mantilla es coherente con la edad límite encuestada que fue 49 años y 50 años para el presente estudio.

En el dominio de tiempo libre se reportó $49 \%$ de inactividad física en tanto que Gómez (25) reportó 56,3 \% y Mantilla 59 \%, explicado de igual manera por el rango de edad incluido por los diferentes estudios, Gómez tomó personas entre 18 y 29 años y Mantilla entre 15 y 49 años.

En el dominio de la actividad física como medio de transporte, el estudio reportó 55,7\% de inactividad física y Mantilla $60 \%$ (11), relacionado con la alta tendencia del estrato 3 a utilizar el transporte público y el automóvil en el estrato 4 .

En el dominio del trabajo se encontró $32 \%$ de inactividad física. Otros estudios reportaron $72,3 \%$ (5) y $55 \%$ (11) prevalencias que resultan elevadas 
en comparación con la encontrada, y se explica en que el $86 \%$ de las personas se dedicaron los últimos 7 días a trabajar, lo que corresponde con el predominio de la clase trabajadora del estrato 3 y contributiva incluida en el estudio. Mantilla (11) encuestó la localidad de Teusaquillo en donde predomina el estrato 4 seguida del 3, lo cual explica la mayor prevalencia, dado que las personas con mejor posición económica realizan trabajos que demandan menor esfuerzo físico. En consecuencia, se debe promover el uso de la bicicleta y la caminata en ésta población dada su elevada inactividad física en éste dominio.

Con relación al sexo, la OMS (4) y algunos estudios han reportado a los hombres como más activos que las mujeres $(2,4,5,11,14)$ lo cual coincide con los resultados de nuestro estudio que encontró a nivel global mayor prevalencia de inactividad física en las mujeres $(58 \%)$ e igualmente en los dominios a excepción de la actividad física en el hogar. Este comportamiento ha sido atribuido por algunos autores al rol de género que se encuentra inmerso en las normas sociales y culturales (11). Por otra parte, se encontró ser mujer asociado inversamente a la práctica de actividad física en el dominio del trabajo $(\mathrm{OR}=0.67)$, lo que sugiere la necesidad de enfocar acciones hacia las mujeres con estrategias que propicien ambientes promotores de actividad física en los dominios trabajo y hogar, por ser un escenario culturalmente reconocido como propio.

Con relación a la edad, el estudio muestra que los adultos entre 41 y 50 años tienen mayor prevalencia de inactividad física, lo que ha sido señalado también por otros autores $(2,6,13,17)$. Se asoció la mayor actividad física a edades entre 25 y 35 años, y entre 36 y 40 años $(O R=1,5$ y $O R=1,7$ respectivamente) comparado con individuos entre 41 y 50 años. Particularmente, en el dominio del hogar se encontró que los menores de 40 años tienen menor probabilidad de ser activos respecto a los individuos entre 41 y 50 años $(\mathrm{OR}=0,57$ y $\mathrm{OR}=0,73)$. Lo anterior sugiere la necesidad de estimular en los menores de 40 años la práctica de la actividad en los dominios diferentes al hogar, lo cual incluye estrategias en su que hacer laboral.

Respecto al estado civil se encontró mayor prevalencia de inactividad física en las personas con pareja estable, lo cual también se observó en los dominios transporte y tiempo libre. En el primero, además se encontró asociado tener pareja estable $(\mathrm{OR}=0,04)$ con una menor probabilidad de ser activo. En tiempo libre, se encontró asociado a no tener una pareja estable con una mayor probabilidad de ser activos $(\mathrm{OR}=1,6)$, contrario a lo encontrado en Perú (26) en donde la actividad deportiva regular se asoció con estado civil casado/ 
conviviente. Esto sugiere la necesidad de estudiar el efecto del apoyo social inmediato -pareja y familia- para involucrarlos en la actividad física.

Con relación al nivel educativo, se encontró asociación entre el nivel de estudios y ser activo en el dominio del hogar, así las personas con nivel educativo ninguno/preescolar, y secundaria tuvieron 5,9 veces y 1,4 veces más probabilidad de ser activos que las personas con formación superior/universitario y postgrado. Igual resultado encontró Mantilla (11). En contraste, en el dominio de tiempo libre se encontró asociado el nivel de estudios primaria con una menor probabilidad de ser activo respecto al superior/universitaria y postgrado $(\mathrm{OR}=0.348)$. Estehallazgo también fue documentado en Brasil (16). Lo anterior, implica diferencias ocupacionales determinadas por el nivel educativo que hace que las personas con menor nivel educativo se ocupen en actividades del hogar y las de mayor nivel en actividades de tiempo libre, lo cual se puede utilizar en estrategias promocionales diferenciadas.

En cuanto a la ocupación de las personas en la última semana, otros estudios reportaron más prevalente la inactividad física en quienes se dedicaron a buscar trabajo $(5,12)$, estudiar (5) y realizar labores del hogar (12). De manera similar, el estudio encontró que las personas inactivas tienen como principal ocupación oficios del hogar, buscar trabajo y ser pensionado o jubilados, categorías que se encontraron asociadas en el dominio del trabajo $(\mathrm{OR}=0,3)$. En éste mismo dominio se encontró asociación en las personas que se dedicaron a trabajar, $\mathrm{y}$ trabajar y estudiar frente a los que estudian solamente $(\mathrm{OR}=9,0$ y $\mathrm{OR}=1,8$ respectivamente). En el dominio del hogar, la práctica de actividad física se asoció con los oficios del hogar $(\mathrm{OR}=3,1)$. En el dominio de tiempo libre se encontró mayor prevalencia de inactividad física entre las personas que trabajaron $(53,2 \%) y$, trabajaron y estudiaron $(51,7 \%)$ sin que exista asociación en éste dominio ni en el dominio transporte. Derivado de lo anterior surge la necesidad de promover entre las personas dedicadas a oficios del hogar, buscar trabajo y pensionados y jubilados la práctica de actividad física.

En lo relacionado con el estado de salud, algunos estudios han reportado la auto-percepción del estado de salud buena o muy buena asociada a la actividad física $(8,11,12)$, acorde con los resultados del estudio que encontró mayor prevalencia de inactividad física en los estados de salud regular y deficiente, y como factor asociado la auto-percepción del estado de salud muy bueno $(\mathrm{OR}=1,6)$ respecto a los estados de salud regular y deficiente. Igual resultado se encontró en los dominios de la actividad física como medio de transporte $(\mathrm{OR}=2,7)$ y $\operatorname{hogar}(\mathrm{OR}=4,9)$. 
Con relación al hábito de fumar, el estudio encontró mayor prevalencia de inactividad física en los no fumadores $(47,9 \%$ ) y asociación con menor actividad física global $(\mathrm{OR}=0,66)$ y en el dominio trabajo $(\mathrm{OR}=0,64)$ contrario al dominio de tiempo libre en donde no fumar se asoció con ser activo $(\mathrm{OR}=1,5)$. En esta asociación pueden incidir variables no contempladas en el estudio por lo que se requiere mayor investigación. En lo referente al consumo de bebidas alcohólicas no se encontró relación entre éste factor y la actividad física.

En cuanto a las variables de contexto barrial, en el dominio de tiempo libre se encontró asociación con la variable de soporte social y la observación de personas físicamente activas en el barrio $(\mathrm{OR}=2,1)$. Otro estudio (8) encontró que las personas activas para la caminata tuvieron más soporte social que los caminantes ocasionales y mucho más que los que nunca caminaban. En contraste, Mantilla (11) encontró asociado de manera inversa la percepción de inseguridad durante la noche, en tanto que en el estudio no se encontró asociación. También se encontró asociada de manera inversa la presencia de tráfico en las calles que dificulta caminar, así las personas que estuvieron parcialmente de acuerdo o en desacuerdo, tuvieron menor probabilidad de ser activas respecto a las que estuvieron completamente en desacuerdo. Otro estudio halló (26) como barrera frecuente para la práctica del deporte y caminata la falta de infraestructura para tal fin, lo cual también ya ha sido documentado por la OMS (17) y descrito también por Eyler (8) en caminantes que reportaron mayor uso de las calles del vecindario, de los espacios internos y externos de centros comerciales y de los parqueaderos cercanos a su vivienda. Lo anterior sugiere la necesidad de modificar el contexto cercano del individuo en términos de infraestructura, así como la importancia del soporte social en tanto factor contribuyente a la práctica de la actividad física.

Como limitación del estudio se debe tener en cuenta que la información utilizada corresponde a una muestra representativa únicamente del estrato tres y afiliada a régimen contributivo de Bogotá •

\section{REFERENCIAS}

1. U.S. Department of Health and Human Services, Centers for Disease Control and Prevention. Physical Activity and Health. A Report of the Surgeon General Journal [Internet]. 1996. Disponible en http//www.cdc.gov/nccdphp/sgr/ Consultado en Enero 2010.

2. Rojas ME, Vélez H, Flórez LE. Actividad física y salud: Una síntesis histórica, epidemiológica y psicosocial. Tipica, Boletín Electrónico de Salud Escolar. 2009 Julio-Diciembre; 5 (2).

3. Vuori I. Physical inactivity as a disease risk and health benefits of increased physical activity. Perspectives Health Enhancing Physical Activity. 2004:6. 
4. WHO. Noncommunicable diseases and mental health noncommunicable disease prevention and health promotion. Health and Development Through Physical Activity and Sport. WHO/NMH/NPH/PAH/03.2; 2003.

5. ICBF, Profamilia. Actividad Física. En: Villegas CB (Ed.). Encuesta Nacional de Situación nutricional en Colombia, ENSIN 2005. Primera ed. Bogotá D. C.; 2006. p. 353-77.

6. OPS. Estrategia Mundial sobre Alimentación Saludable, Actividad Física y Salud (DPAS). Plan de Implementación en América Latina y el Caribe. 2006-2007. DPC/NUT/001-06. Washington D.C.; 2006.

7. IEDAR [Internet] Actividad física Factor clave en la prevención de la obesidad. Disponible en: http://www.iedar.es/nuevo/act_fisic.pdf. Consultado en Mayo de 2010.

8. Eyler AA, Brownson RC, Bacak SJ, Housemann RA. The epidemiology of walking for physical activity in the United States. Med Sci Sports Exerc. 2003 Sep; 35(9):152936.

9. Cabrera G, Gómez L, Mateus JC. Actividad física y etapas de cambio comportamental en Bogotá. Colombia Médica. 2004; 35(2):82-6.

10. Ministerio de la Protección Social, Colciencias, Agudelo CA, Prieto A. Modelos de Movilización social con énfasis en actividad física y estilos de vida saludables para reducir el sedentarismo en las regiones de Bogotá D.C., Antioquia y Quindío. Bogotá D.C.: Universidad Nacional de Colombia; 2006.

11. Mantilla S. Actividad Física en Habitantes de 15 a 49 Años de una Localidad de Bogotá, Colombia, 2004 . Rev Salud pública (Bogotá). 2006; 8(Sup. 2):69-80.

12. Gómez LF, Duperly J, Lucumí DI, Gámez R, Venegas AS. Nivel de actividad física global en la población adulta de Bogotá (Colombia). Prevalencia y factores asociados. Gac Sanit. 2005;19(3):206-13.

13. Bauman A, Bull CF, Craig CL, Ainsworth BE, Sallis JF, Bowles HR, et al. The International Prevalence Study on Physical Activity: results from 20 countries. International Journal of Behavioral Nutrition and Physical Activity. 2009; 6(21):1-11.

14. Gómez LF, Mateus JC, Cabrera G. Leisure-time physical activity among women in a neighbourhood in Bogotá, Colombia: prevalence and socio-demographic correlates. Cad Saúde Pública. Rio de Janeiro. 2004; 20(4):1103-9.

15. Pate RR, Pratt M, Blair SN, Haskell WL, Macera CA, Bouchard C, et al. Physical activity and public health. A recommendation from the Centers for Disease Control and Prevention and the American College of Sports Medicine. JAMA. 1995; 273(5):402-7.

16. Salles-Costa R, Werneck L, Lopes S, Faerstein E. Associação entre fatores sóciodemográficos e prática de atividade física de lazer no Estudo Pró-Saúde. Cad Saúde Pública. Rio de Janeiro. 2003 jul-ago;19(4):1095-105.

17. WHO. Health and Development Through Physical Activity and Sport. WHO/NMH/NPH/PAH/ 03.2; 2003.

18. Prieto A, Agudelo CA. Enfoque multinivel para el Diagnóstico de la Actividad Física en tres regiones de Colombia. Revista de Salud Pública (Bogotá). 2006;8(Sup.2):57-68.

19. IPAQ. Guidelines for Data Processing and Analysis of the International Physical Activity Questionnaire (IPAQ) - Short and long Forms. Journal [Internet]. 2005: Disponible en: http://www.ipaq.ki.se Consultado: Mayo de 2007.

20. OPS. Estrategia Mundial sobre Alimentación Saludable, Actividad Física y Salud (DPAS). Plan de Implementación en América Latina y el Caribe. 2006-2007. Journal [serial on the Internet]. 2006. Disponible en: http://www.paho.org/spanish/ad/dpc/nc/dpasplan-imp-alc.pdf Consultado: Diciembre 2010.

21. William H, I-Min L, Russell P, Kenneth E. Powell N, Blair F., et al. Physical Activity and Public Health. Updated Recommendation for Adults From the American College of Sports Medicine and the American Heart Association. Special Reports. Official Journal of the American College of Sports Medicine. 2007;1423-34.

22. Londoño JL. Análisis multivariado. Metodología de la investigación epidemiológica. 3ra ed. Bogotá: El Manual Moderno; 2004. p. 177-95. 
23. Silva LC. Excursión a la regresión logística en ciencias de la salud. Madrid, España: Ediciones Díaz de Santos S.A.; 1995.

24. Hosmer D, Lemeshow S. Applied Logistic Regression. Second ed. Estados Unidos: Jhon Wiley and sons, Inc.; 2000.

25. Gómez LF, Lucumí DI, Girón SL, Espinosa G. Conglomeración de Factores de riesgo de comportamiento asociados a Enfermedades crónicas en adultos jóvenes de dos localidades de Bogotá, Colombia: Importancia de las diferencias de género. Rev Esp Salud Pública. 2004;78(4):493-504.

26. Seclén-Palacín JA, Jacoby ER. Factores sociodemográficos y ambientales asociados con la actividad física deportiva en la población urbana del Perú. Rev Panam Salud Publica/Pan Am J Public Health. 2003;14(4):255-64. 